\title{
Determination of the Temperature-Dependent Thermal Material Properties in the Cooling Process of Steel Plates
}

\author{
Dimitri Rothermel $\mathbb{D}^{1},{ }^{1}$ Thomas Schuster $\mathbb{D}^{1},{ }^{1}$ Roland Schorr $\mathbb{D}^{2},{ }^{2}$ and Martin Peglow $\mathbb{1 D}^{2}$ \\ ${ }^{1}$ Department of Mathematics, Saarland University, Saarbrücken, Germany \\ ${ }^{2}$ Research \& Development, AG der Dillinger Hüttenwerke, Dillingen, Saar, Germany \\ Correspondence should be addressed to Thomas Schuster; thomas.schuster@num.uni-sb.de
}

Received 6 October 2020; Revised 10 February 2021; Accepted 12 February 2021; Published 8 March 2021

Academic Editor: Bekir Sahin

Copyright ( 2021 Dimitri Rothermel et al. This is an open access article distributed under the Creative Commons Attribution License, which permits unrestricted use, distribution, and reproduction in any medium, provided the original work is properly cited.

\begin{abstract}
Accelerated cooling (ACC) is a key technology in producing thermomechanically controlled processed (TMCP) steel plates. In a TMCP process, hot plates are subjected to a strong cooling resulting in a complex microstructure leading to increased strength and fracture toughness. The microstructure, residual stresses, and flatness deformations are strongly affected by the temperature evolution during the cooling process. Therefore, the full control (quantification) of the temperature evolution is essential regarding plate design and processing. It can only be achieved by a thermophysical characterization of the material and the cooling system. In this paper, the focus is on the thermophysical characterization of the material properties which govern the heat conduction behavior inside of the plates. Mathematically, this work considers a specific inverse heat conduction problem (IHCP) utilizing inner temperature measurements. The temperature evolution of a heated steel plate passing through the cooling device is modeled by a $1 \mathrm{D}$ nonlinear partial differential equation with temperature-dependent material parameters which describe the characteristics of the underlying material. Usually, the material parameters considered in IHCPs are often defined as functions of the space and/or time variables only. Since the measured data (the effect) and the unknown material properties (the cause) depend on temperature, the cause-to-effect relationship cannot be decoupled. Hence, the parameter-to-solution operator can only be defined implicitly. By proposing a parametrization approach via piecewise interpolation, this problem can be resolved. Lastly, using simulated measurement data, the presentation of the numerical procedure shows the ability to identify the material parameters (up to some canonical ambiguity) without any a priori information.
\end{abstract}

\section{Introduction}

This paper considers the initial-boundary-value problem (IBVP)

$$
\begin{aligned}
C(u) u_{t} & =\left(k(u) u_{z}\right)_{z}, \quad(t, z) \in I \times \Omega, \\
u & =u^{b}, \quad t \in I, z=0, \\
u & =u^{t}, \quad t \in I, z=L, \\
u & =u_{0}, \quad t=0, z \in \Omega,
\end{aligned}
$$

in order to model the heat conduction part of the cooling process inside of heavy plates made of steel. For $L, T>0$, $z \in \Omega:=(0, L)$ and $t \in I:=[0, T]$ represent the space and time variable, respectively. The solution $u(t, z)$ of the IBVP is the temperature and $u_{0}$ is the known initial temperature distribution. The gathering of the Dirichlet boundary conditions $u^{b}$ and $u^{t}$ from real thermocouple measurements will be explained later. Given some additional temperature state $u^{c}$ at the core $z=L / 2$, the aim of the paper is to solve the inverse heat conduction problem (IHCP) in identifying the temperature-dependent thermal material properties, especially the thermal conductivity $k$ and the volumetric heat capacity $C$. In the following, these thermal properties will be referred to as material parameters.

Such IHCPs belong to the class of inverse heat transfer problems (IHTPs) which by themselves are studied extensively in the current literature. For general information on 
IHTPs, the interested reader refers to the standard textbooks as $[1,2]$ or [3]. It is worth mentioning that the IHCPs in this paper are not to be confused with inverse heat convection problems which are often presented in the literature (see e.g., [4-6]). There, the goal is to determine some information about the boundary flux conditions, e.g., Neumann or Newton boundary conditions which are convective type.

This paper is motivated in the manufacturing process of high-strength low-alloy water-cooled heavy plates, where the material properties are the key targets. Customer requirements for various macroscopic material properties are achieved by specific microstructures. Water cooling processes are an important instrument for this specific adjustment of the microstructure, cf. [7, 8]. These cooling processes however generate residual stresses that can have an unfavorable effect on the flatness of the products. In order to still be able to meet the high demands on material properties and flatness of the plates, a deep understanding and targeted control of cooling processes on an industrial scale is imperative, cf. [9-11].

Modern cooling systems today allow very precise temperature control, which is essential for the targeted adjustment of the material properties and is limited only by the physics of heat conduction. Regarding the automation of such a cooling system, it is not sufficient to ensure compliance with the strategy parameters, such as the start and end cooling temperature at defined control times, using simple precontrol concepts. Instead, a modern precontrol concept must be able to implement the strategy parameters that result from complex temperature control under production conditions.

From a thermal point of view, cooling processes consist on the one hand of an interaction between the cooling water and the hot plate surface (heat convection) and on the other hand of a heat transport within the plate (heat conduction), in particular through the plate thickness. The thermophysical variables that characterize heat transfer and heat conduction are temperature-dependent variables. In addition, there are other influencing factors that are necessary to fully characterize them. While the heat convection is strongly dependent on the surface structure and the cooling medium or its condition, the heat conduction within the plate is influenced by its chemical analysis and the underlying microstructure.

The first question that arises is how the two processes of heat conduction and heat transfer, which occur simultaneously in a cooling process, can be studied separately for the purpose of modeling. In this paper this question is answered by using the measurement results of a full-scale experiment, i.e., a production cooling, to model the heat conduction alone.

Subsequently, the question arises on which scale the modeling has to be carried out in order to guarantee a production-oriented application. The focus is on the complexity of the underlying model and the number of its degrees of freedom. Any possible answer to this question must be considered in the context that, in the production of heavy plates, a wide range of alloying compositions and a multitude of structural variations are used which have a strong influence on the heat conduction behavior. Therefore, in this paper, a class formation regarding chemical analysis and microstructure variation is assumed, so that the heat conduction in the respective class is only a function of the temperature.

More specifically, the temperature inside of the plate is solely driven by its material parameters, i.e., the volumetric heat capacity and the thermal conductivity, which are denoted by $C$ and $k$, respectively. Often, the literature contains various models where the material parameters only depend on the space variable $z[12,13]$ or only on the time variable $t[14]$. The estimation of temporally and spatially varying diffusion coefficients is considered in [15] (see also the references contained therein).

However, in the 1D heat conduction, the PDE model considered in this article is given as

$$
C(u) u_{t}=\left(k(u) u_{z}\right)_{z}
$$

which will be discussed in Section 3 in more detail; the material parameters depend on the temperature $u$ itself due to the high temperature gradients in the material. Note that 5 is often also used in the modeling of heating processes, i.e., the addition of heat, e.g., in simulations of blast furnaces or in space research. Yet, in this paper, the interest is in the cooling process, i.e., the extraction of heat from heated heavy plates made of steel by applying cooling water on the surfaces.

The current literature proposes simple numerical tricks in order to avoid the temperature dependency of the material parameters. In [16] or [17], for example, the authors model the material parameters as low-order polynomials in temperature and show that they are able to identify the associated coefficients. This way, in order to represent more complex functions one needs to increase the order of the polynomial significantly, which has its own drawbacks as numerical instabilities arise due to fact that the coefficients of the high order monomials might be very small. In [18], Huang and Jan-Yuan also try to determine more complex material parameters $k(u)$ and $C(u)$ simultaneously by arguing that, once the temperature distribution $u(t, z)$ is known, one can replace $k(u)$ by $k(t, z)$ and $C(u)$ by $C(t, z)$. Yet again, using a numerical scheme, the determination of some time and space-dependent material parameters gets computationally more expensive when refining the spatial and temporal grid size which indeed is also a drawback. That is why this paper proposes a method to solve simultaneously for general functions $k(u)$ and $C(u)$ in the space $\mathscr{C}^{1}$ of continuously differentiable functions with no a priori information and without restricting the form of the functions too drastically by, e.g., using a polynomial representation.

Nevertheless, concerning the inverse heat conduction problem (IHCP), circumventing the temperature dependency of the material parameters is not a bad idea not just because of reasons regarding the numerical implementation. Typically, one formulates a classical inverse problem by providing the forward operator 


$$
\begin{gathered}
F: X \longrightarrow Y, \\
x \mapsto y,
\end{gathered}
$$

which maps a parameter $x \in X$ to some solution $y \in Y$. Depending on the problem, a forward computation of the solution $y$ requires the calculation of a parameter- $x$-dependent integral, a boundary-value problem, or even an initial-boundary-value problem. For the inverse problem the solution, $y$ is assumed to be known, while the parameter $x$ has to be identified. Instead of $y$, one often observes or measures a noisy version $y^{\delta}$ of the exact solution, where

$$
\left\|y^{\delta}-y\right\|_{Y} \leq \delta
$$

and $\delta>0$ is the so-called noise level. Thus, the solution of the inverse problem becomes the parameter $x$ of the abstract operator equation

$$
F(x)=y^{\delta},
$$

although the measured data $y^{\delta}$ does not have to belong to the image space $Y$. A common way is then to declare the solution of the inverse problem as the minimizer of the objective functional

$$
\min _{x \in X}\left\|F(x)-y^{\delta}\right\|_{Y}^{2}
$$

Not relevant for this paper, but depending on the problem, the minimization process of (10) might require regularization techniques, cf. $[19,20]$. Note that, in a usual scenario such as in (9), the parameter-to-solution relationship can be formulated explicitly by the forward operator $F$. In this paper, the aim is to determine the temperature-dependent material parameters $C(u)$ and $k(u)$ given temperature measurements, which can be denoted by $u$ at this point (one assumes for a moment that the full temperature field can be measured without measurement errors; “ " stands for "corresponds to"):

$$
\begin{aligned}
x & \sim\{k(u), C(u)\} \\
y^{\delta} & \sim u .
\end{aligned}
$$

Because the material parameters and the measurement data both depend on the temperature outcome (the temperature outcome is the full data in this example), which depends on the material parameters itself, i.e., $u=u(k, C)$, a classical formulation of the forward operator is not possible, because the parameter-to-solution relationship cannot be decoupled. A forward operator could only be posed implicitly, for example, with an appropriate definition by

$$
F(k(u), C(u), u)=0
$$

To be able to formulate the above IHCP in a classical sense, one proposes a convenient parametrization approach of the material parameters using piecewise cubic interpolation. This allows the decoupling of the parameter-to-solution relationship, yielding an explicitly defined forward operator

$$
F: \underline{p} \longrightarrow u
$$

where $p$ represents the material parameters $k(u)$ and $C(u)$ without depending on the temperature $u$ anymore. Again, the highlight is that one will be still able to identify general functions $k(u)$ and $C(u)$ in the space $\mathscr{C}^{1}$, cf. Section 5 (the identification is up to some canonical ambiguity).

Still, the focus of this paper is application-oriented. After introducing the experimental setup in Section 2 and the mathematical description in Section 3 and 4, a practical guide in Section 5 on how to numerically implement the underlying parameter estimation method is shown.

\section{Experimental Setup}

More specifically, this paper is motivated in the modeling of a MULPIC (MULti-Purpose Interrupted Cooling, cf. $[21,22])$ laminar cooling process for cooling low-alloy heavy plates (C-content about $0.10 \% \mathrm{C}$ ) with typical start and end cooling temperatures between 750 and $850^{\circ} \mathrm{C}$ and 400 and $550^{\circ} \mathrm{C}$ respectively.

In order to understand the underlying laminar cooling system, the reader is referred to the sketch in Figure 1. Multiple conveyor rollers transport the heated heavy plate in $x$-direction towards the cooling system, consisting of several water cooling zones that are indicated by dots.

Depending on the material parameters (thermal conductivity, volumetric heat capacity), applying cooling water on top and bottom surfaces (with respect to the $x$-y-plane) leads to a special heat transfer within the plate and thus to a very specific final temperature profile.

Typically, the heavy plates under consideration have a very small thickness in comparison to their length and width. Figure 2 refers to a schematic representation of the heavy plate dimensions. Therefore, the main information about the temperature evolution while cooling lies in the heat transfer in $z$-direction.

Hence, in order to gather the main heat conduction information, measured temperatures at different depths with respect to the thickness of the heavy plate are needed. This is achieved by placing three so-called thermocouples into the heavy plate, indicated by the red dots in Figure 2 . We note that, in this figure, the red dots represent the hot junction of the thermocouples and not a cross section of blast-holes. In the experimental setup, the thermocouples are positioned right in the middle with respect to the width and also symmetrically with respect to the thickness, i.e., one has one center thermocouple and two near-surface thermocouples, one at the top and one at the bottom.

The experimental setup is chosen to simulate real production conditions. From a process engineering point of view, this means that the dimensions of the test plate must be chosen appropriately so that stable flow conditions on the upper side of the plate are established during the cooling process, i.e., during the measurement. The temperature measurements used here are therefore based on plate 


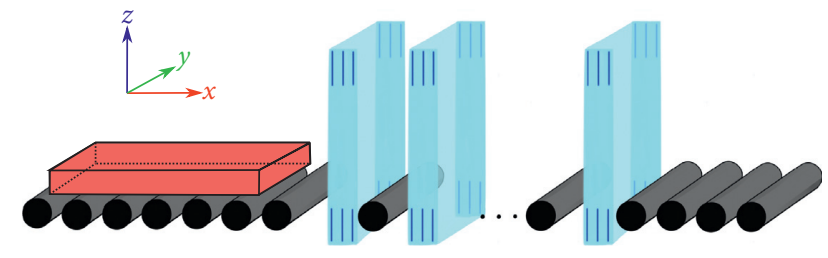

FIGURE 1: Sketch of the laminar cooling system.

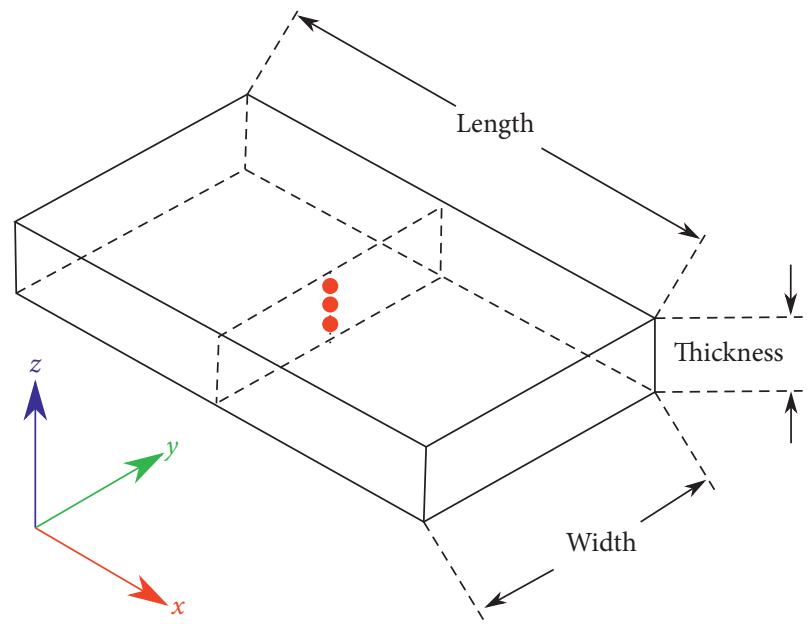

Figure 2: Placement of thermocouples into the heavy plate indicated by the red dots.

dimensions with widths between 2.0 and $4.5 \mathrm{~m}$, lengths between 10 and $16 \mathrm{~m}$ and a thickness of $45 \mathrm{~mm}$. A measuring probe is installed in the middle of these test plates with regard to length and width. This means that the material is cut out by flame in the middle of the plate and instrumented with type $\mathrm{K}$ thermocouples with a diameter of $1.5 \mathrm{~mm}$. The thermocouples inserted in lateral holes have to be welded at the exit point to fix them. To be able to record their signal under water, a $10 \mathrm{~Hz}$ data logger is being installed in a thermobox on the test plate. In order to not to disturb the flow conditions during the cooling process, a minimum distance of approximately $5 \mathrm{~m}$ between measuring probe and thermobox has to be considered. The measuring signal is then transmitted via a supply line to be installed in a masked groove between probe and logger.

Figure 3 shows a detailed section of the marked cross section in Figure 2. The thermocouples are color-coded in such a way that one can directly assign the measured temperature curves in Figure 4 to its corresponding depth by color. The variable $L>0$ is the length of the distance between the top and bottom thermocouple. By applying cooling water evenly on top $(z>L)$ and bottom surface $(z<0)$ of the heavy plate, one can assume that, for some fixed depth $0<z<L$, the temperatures are equal with respect to width at any time.

In Figure 4, the measured temperatures $u^{t}, u^{c}$, and $u^{b} \in \mathbb{R}_{+}^{m}(m>0$, the subscripts refer to the positions top, center and bottom) from a real scenario are plotted as

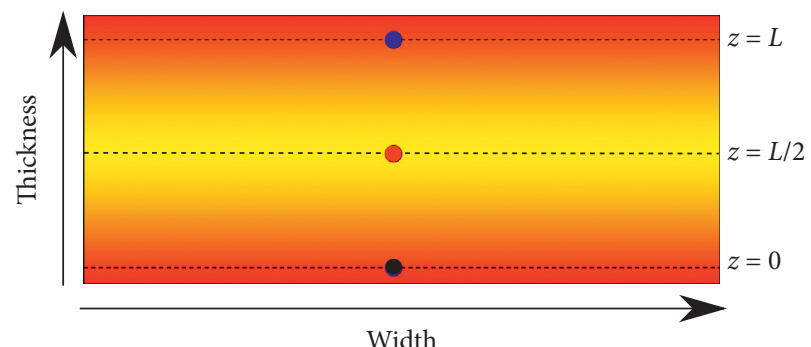

Figure 3: Color-coded thermocouples at different depths $z$.

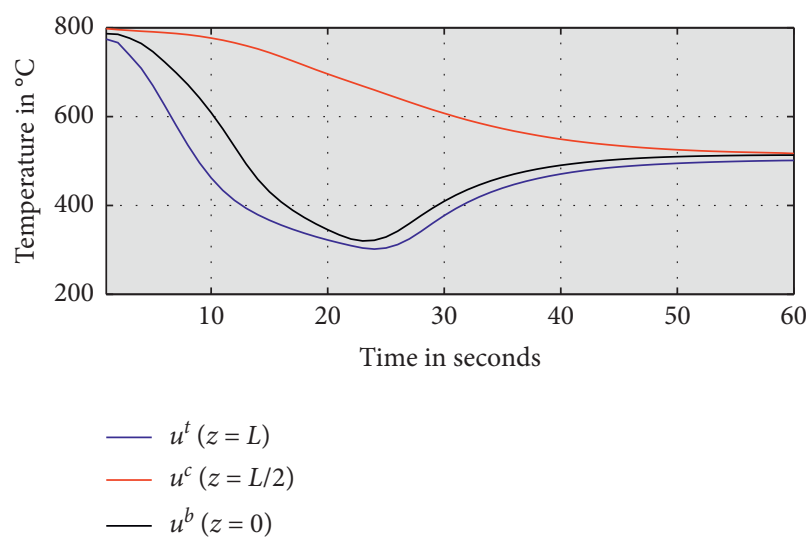

FIgURE 4: Measured temperatures at different thermocouple locations.

interpolated curves. The number $m>0$ depends on the frequency of the built-in data logger which records the history of a typical cooling scenario of such plates as follows:

(i) Before the cooling, at time $t=0[s]$, the heavy plate is almost evenly heated at approx. $800^{\circ} \mathscr{C}$.

(ii) During the water cooling process $(0<t<25[s])$, the temperature decreases whereas the cooling rate of the near-surface regions is larger than the cooling rate of the core region due to the smaller distance to the cooled surfaces.

(iii) When leaving the cooling system, the cooling process is getting interrupted and recovery starts, at times $t>25[s]$. Due to heat conduction under air cooling conditions, heat flows from the core region to the near-surface regions which can be seen from an increase of temperature (see $u^{t}$ and $u^{b}$ ).

The exact dependence of the temperature on space and time is highly affected by the material parameters. In the following, these material parameters will be determined as a solution of an inverse heat conduction problem, where the measured temperatures $u^{t}$ and $u^{b}$ are used for the Dirichlet boundary conditions of the PDE model. The measured core temperature $u^{c}$ is left to be the data, see Section 4 and 5 .

\section{Mathematical Preliminaries}

Before formulating the inverse heat conduction problem in order to determine the temperature-dependent material 
parameters, it is necessary to specify which mathematical model is used. Additionally, it is needed to define the socalled parameter-to-solution operator and the observation operator.

3.1. Model Description. As already mentioned in Section 2, the heavy plates under consideration have a very small thickness compared to their length and width. Cooling of top and bottom surfaces of such plates results in a 1D heat conduction problem. The temperature evolution of every 2 points of this 1D object includes the thermophysical information of the regime in between these 2 points.

For $L>0$, let $\Omega:=(0, L)$ contain the spatial variables $z$ which describe the positions/depths in the heavy plate in direction of thickness, while $z=0$ corresponds to the position of the bottom and $z=L$ to the position of the top thermocouple (Figure 3).

The 1D model for heat conduction in the time frame $I:=(0, T]$ for some $T>0$ is then given by the initialboundary-value problem:

$$
\begin{aligned}
\widetilde{C}(u) u_{t} & =\left(\widetilde{k}(u) u_{z}\right)_{z}, \quad(t, z) \in I \times \Omega \\
u & =u^{b}, \quad t \in I, z=0, \\
u & =u^{t}, \quad t \in I, z=L, \\
u & =u_{0}, \quad t=0, z \in \Omega,
\end{aligned}
$$

where the unknown solution $u: \bar{I} \times \bar{\Omega} \longrightarrow \mathbb{R}_{+}$represents the temperature at times $t \in[0, T]$ and depths $z \in[0, L]$. The function $u_{0}(z)$ is the known initial temperature distribution before cooling, i.e., for $t=0$. For the Dirichlet boundary conditions (16)-(17), the use of the measured boundary temperatures $u^{b}$ and $u^{t}$ are proposed (Figure 4$)$. For brevity, sometimes the variables $t, z$, or $(t, z)$ are omitted, e.g., $u=u(t, z)$. By the subscripts $t$ and $z$, one refers to the derivatives $\partial / \partial t$ and $\partial / \partial z$ with respect to time and space, respectively.

Let $U=\left[u_{\min }, u_{\max }\right]$ with $0 \leq u_{\min }<u_{\max }<\infty$ be the interval that covers all occurring temperatures $u$ in (15)-(18). In the case of the cooling of heated heavy plates, the maximum temperature of the plates is at the start of the cooling process. Also, the temperatures do not fall below zero or the temperature of the cooling water. Thus, one can set, e.g.,

$$
u_{\min }=0 \text { and } u_{\max }=\max _{z \in \bar{\Omega}} u_{0}(z) .
$$

The functions

$$
\begin{aligned}
& \tilde{C}: U \subset \mathbb{R}_{+} \longrightarrow \mathbb{R}_{+}, \\
& \tilde{k}: U \subset \mathbb{R}_{+} \longrightarrow \mathbb{R}_{+},
\end{aligned}
$$

denote the volumetric heat capacity and the thermal conductivity, respectively. Let us assume that $\widetilde{k}, \widetilde{C} \in \mathscr{C}^{1}(U)$. These functions represent the material parameters which lead to a unique heat conduction behavior and consequently to a very specific temperature solution $u(t, z),(t, z) \in \bar{I} \times \bar{\Omega}$. Both functions depend on $u \in \mathbb{R}_{+}$itself due to the presence of high temperature gradients while cooling. Thus, the 1D heat equation (15) is nonlinear in $u$.

For this paper, the aim is to put the focus on the parameter estimation methodology consisting of the determination of these material parameters $\widetilde{C}$ and $\widetilde{k}$ from core temperature knowledge rather than discussing the mathematical aspects of abstract function spaces and the theory contained therein. For that reason, we want to make the generalized assumption that all functions in (15)-(18) are sufficiently smooth in the sense that the heat equation model is well-defined. Furthermore, we would also like to assume that a unique solution $U \ni u: \bar{I} \times \bar{\Omega} \longrightarrow \mathbb{R}_{+}$always exists. Without specifying the function space $\mathcal{U}$, we note that the point evaluation is valid in time and space, i.e., $u(t, z)$ is at least continuous in $t \in \bar{I}$ and $z \in \bar{\Omega}$. We recommend [23] for more information about the abstract function spaces.

3.2. Parameter-To-Solution Operator. Let $\mathscr{K}$ and $\mathscr{C}$ be the spaces of all admissible functions $\widetilde{k}$ and $\widetilde{C}$ in (15), respectively. Let the operator $\widetilde{S}$ be defined by

$$
\begin{gathered}
\widetilde{S}: \mathscr{K} \times \mathscr{C} \longrightarrow \mathcal{U}, \\
(\tilde{k}, \widetilde{C}) \longmapsto u,
\end{gathered}
$$

where $u \in \mathscr{U}$ is the solution to the initial-boundary-value problem (15)-(18) corresponding to material parameters $(\widetilde{k}, \widetilde{C})$. One faces problems in defining some parameter-tosolution operator as in (22)-(23) explicitly, because the material parameters $\widetilde{k}$ and $\widetilde{C}$ depend on the temperature $u$ which in turn depends on $\widetilde{k}$ and $\widetilde{C}$. It is possible to formulate an implicitly defined parameter-to-solution operator, but that is not furthermore pursued in this article. Rather, the temperature dependency of the input functions is removed without discarding the underlying PDE, i.e., the goal is to retain the heat conduction models (15)-(18) up to some slight changes.

For that reason, let $\pi_{n}: u_{\text {min }}=u_{1}<u_{2}<\cdots<u_{n}=u_{\max }$ be a partition of the interval $U=\left[u_{\min }, u_{\max }\right]$. Moreover, let $\underline{k}=\left(k_{1}, \ldots, k_{n}\right)^{T} \in \mathbb{R}_{+}^{n}$ and $\underline{C}=\left(C_{1}, \ldots, C_{n}\right)^{T} \in \mathbb{R}_{+}^{n}$ be two sets of values. Given $\pi_{n}, \underline{k}$, and $\underline{C}$, construct piecewise cubic functions $k$ and $C$ in $\mathscr{C}^{1}(U)$, such that,

$$
\begin{aligned}
& k\left(u_{i}\right)=k_{i}, \\
& C\left(u_{i}\right)=C_{i},
\end{aligned}
$$

for $i=1, \ldots, n$. Choosing $k$ and $C$ in this way, we guarantee that they are sufficiently smooth and can be controlled by the given function values.

In order to represent the temperature-dependent material parameters $\widetilde{k}$ and $\widetilde{C}$ in a parametric form, one uses the approximation properties of piecewise cubic interpolation methods, i.e., for all $\varepsilon>0$, there exists a number $n$ of partition points, such that 


$$
\begin{aligned}
& \sup _{u \in U}|\widetilde{k}(u)-k(u)|<\varepsilon, \\
& \sup _{u \in U}|\widetilde{C}(u)-C(u)|<\varepsilon .
\end{aligned}
$$

This means that neglecting some small error, $\widetilde{k}(u)$ and $\widetilde{C}(u)$ can be replaced by $k(u)=k(u, \underline{k})$ and $C(u)=C(u, \underline{C})$. Given the fixed partition $\pi_{n}$ and the interpolation method, the functions $k$ and $C$ can be represented only by the parameter vector $(\underline{k}, \underline{C})^{T}$.

With this, the parameter-to-solution operator is explicitly defined by

$$
\begin{array}{r}
S: \mathbb{R}_{+}^{2 n} \longrightarrow \mathcal{U}, \\
\underline{p}:=(\underline{k}, \underline{C})^{T} \longmapsto u,
\end{array}
$$

where $u \in \mathcal{U}$ is the solution to the modified initialboundary-value problem (IBVP)

$$
\begin{aligned}
C(u) u_{t} & =\left(k(u) u_{z}\right)_{z}, \quad(t, z) \in I \times \Omega \\
u & =u^{b}, \quad t \in I, z=0, \\
u & =u^{t}, \quad t \in I, z=L, \\
u & =u_{0}, \quad t=0, z \in \Omega,
\end{aligned}
$$

some material parameters are represented by $(\underline{k}, \underline{C})^{T}$ with known $u^{b}, u^{t}$, and $u_{0}$.

3.3. Observation Operator. While a solution $u \in \mathcal{U}$ for the IBVP (29)-(32) is a function of time $t \in \bar{I}$ and position $z \in \bar{\Omega}$, the available data $u^{c}$ consist of discrete temperature measurements at the center of the heavy plate (Section 2 ). That means the position $z=L / 2$ is fixed and the temperature is recorded at times $t_{j}$ for $j=1, \ldots, m$, i.e., $u^{c}=\left(u_{1}^{c}, \ldots, u_{m}^{c}\right)^{T} \in \mathbb{R}_{+}^{m}$.

To be able to compare the output of the parameter-tosolution operator with the data, an auxiliary operator, the socalled observation operator, is needed. This finally allows one to formulate the mathematical model of the inverse heat conduction problem. The observation operator is given as

$$
\begin{aligned}
Q: & \mathcal{u} \longrightarrow \mathbb{R}_{+}^{m}, \\
& u \longmapsto\left(u\left(t_{1}, \frac{L}{2}\right), \ldots, u\left(t_{m}, \frac{L}{2}\right)\right)^{T},
\end{aligned}
$$

\section{Determining the Material Parameters $C$ and $k$}

4.1. Formulation of the Inverse Heat Conduction Problem. For given functions $u^{b}, u^{t}$, and $u_{0}$ and some given data $u^{c} \in \mathbb{R}_{+}^{m}$, the aim is to deduce the heat conduction behavior, i.e., find the optimal (interpolated) material parameters $k_{\mathrm{opt}}$ and $C_{\mathrm{opt}}$, represented by the function values $\underline{k}_{\mathrm{opt}}$ and $\underline{C}_{\mathrm{opt}}$ to the fixed partition $\pi_{n}: u_{\text {min }}=u_{1}<u_{2}<\cdots<u_{n}=u_{\text {max }}$, such that the observed temperature fits the data to some degree. More precisely, the goal is to determine

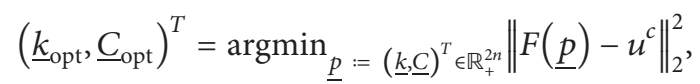

where $F=Q \circ S$ is the forward operator with $Q$ being the observation operator (33) and $S$ is the parameter-to-solution operator (28). Here, the standard euclidean norm is denoted by $\|\cdot\|_{2}$.

Given the data $u^{c} \in \mathbb{R}_{+}^{m}$, one can assume that a solution (34) always exists.

Note that $u^{c} \in \mathbb{R}_{+}^{m}$ contains small measurement errors in the sense that

$$
\sum_{j=1}^{m}\left(u^{e x}\left(t_{j}, \frac{L}{2}\right)-u_{j}^{c}\right)^{2} \leq \delta,
$$

for some $\delta>0$, where $u^{e x}(\cdot, L / 2)$ denotes the time evaluation of the exact temperature $u^{e x}$ at the core position which is of course not available in reality. Nevertheless, the noise level $\delta$ is small, such that $u^{c}$ represents reliable data. Furthermore, a full discretization of the forward operator leads to a finitedimensional range and thus to an inverse problem that is well-posed, but probably ill-conditioned [20]. This means that the underlying inverse problem is not ill-posed in the sense of Nashed [24] and at most mildly ill-conditioned. Hence, small errors in the data amplify the errors in the solution only slightly and a regularizing technique, e.g., by adding a penalty term in (34), is not necessary. Readers who are interested in regularization methods in general settings are referred to [24].

In Section 4.2, an important fact about the ambiguity of solutions is discussed.

4.2. Ambiguous Solution of the Inverse Problem. It is worth mentioning that the solution pair $\left(\underline{k}_{\text {opt }}, \underline{C}_{\text {opt }}\right)^{T} \in \mathbb{R}_{+}^{2 n}$ cannot be the unique minimizer of the least-squares functional:

$$
J(\underline{p}):=\left\|F(\underline{p})-u^{c}\right\|_{2}^{2}
$$

because the forward operator $F$ is not injective, i.e., for any real number $\alpha>0$, it holds that

$$
F(\underline{p})=F(\alpha \underline{p}) \quad \forall \underline{p} \in \mathbb{R}_{+}^{2 n} .
$$

This follows from the fact that multiplying equation (29) by some scalar $\alpha$ does not influence the differential equation. Thus, it is impossible to eliminate the ambiguity of the inverse problem solution. Rather, one has to accept the solution, knowing that only the quotient is uniquely determined:

$$
\lambda_{\mathrm{opt}}(u):=\frac{k_{\mathrm{opt}}(u)}{C_{\mathrm{opt}}(u)} .
$$

This way one can deal with the non-injectivity (37) of the forward operator, because $p=(\underline{k}, \underline{C})^{T}$ and $\alpha p=(\alpha \underline{k}, \alpha \underline{C})^{T}$ are from the same class sharing the same quotient of the corresponding interpolated functions, i.e.,

$$
\frac{k(u)}{C(u)}=\lambda(u)=\frac{\alpha k(u)}{\alpha C(u)} .
$$


In thermodynamics, $\lambda$ is the so-called thermal diffusivity of the material.

Here, the thermal conduction behavior of the material depends mainly on the thermal diffusivity $\lambda$. A perfectly decoupled characterization of $k$ and $C$ is not possible. Interestingly, this coincides with the insights for the solution of linear inverse heat conduction problems where only the scalar thermal diffusivity is discussed.

Remark: in order to be able to identify a heat flux $q(t)$ with Fourier's law of heat conduction in the form of

$$
\pm k(u) u_{z}=q \text {, }
$$

on the surfaces of the heavy plate, the knowledge of the functional form of $k(u)$ is needed. However, the identification of some solution pair $(\alpha \underline{k}, \alpha \underline{C})^{T}$ is appropriate, if one accepts that the determination of $q$ is only disturbed by scalar and time-independent factors $\alpha$, i.e.,

$$
\begin{aligned}
\pm \alpha k(u) u_{z} & =\tilde{q}, \\
q & =\frac{\tilde{q}}{\alpha} .
\end{aligned}
$$

This is the main reason why this paper proposes a method in order to simultaneously determine both the functional forms of $k$ and $C$.

The numerical implementation of solving the parameter estimation problem (34) is discussed in Section 5.

\section{Implementation Approach and Numerical Results}

While the previous sections were more introductory and theoretical, in this section, a practical guide is given on how to numerically solve the parameter estimation problem, i.e., the minimization

$$
\min _{\underline{p} \in \mathbb{R}_{+}^{2 n}} J(\underline{p})=\min _{\underline{p} \in \mathbb{R}_{+}^{2 n}}\left\|F(\underline{p})-u^{c}\right\|_{2}^{2} .
$$

Assuming that $M \geq 1$ experiments were recorded, one can even generalize the objective functional to

$$
J_{M}(\underline{p})=\sum_{i=1}^{M}\left\|Q^{\circ} S_{i}(\underline{p})-u^{c, i}\right\|_{2}^{2},
$$

where $S_{i}$ maps the parameter vector $p=(\underline{k}, \underline{C})^{T}$ to the solution of the initial-boundary-value problem:

$$
\begin{gathered}
C(u) u_{t}=\left(k(u) u_{z}\right)_{z},(t, z) \in I \times \Omega, \\
u=u^{b, i}, \quad t \in I, z=0, \\
u=u^{t, i}, \quad t \in I, z=L, \\
u=u_{0}^{i}, \quad t=0, z \in \Omega,
\end{gathered}
$$

for $i=1, \ldots, M$. Note that the minimization (43) represents the special case $M=1$. Recording and using several experiments stabilize the minimization process and yield better results in case of noisy core measurements $u^{c, i}$.

In this section, the following topics are addressed:

(a) Representation of temperature-dependent material parameters $k(u), C(u) \in \mathscr{C}^{1}(U)$ by some parameter vector $\underline{p}=(\underline{k}, \underline{C})^{T} \in \mathbb{R}_{+}^{2 n}$.

(b) Implementation of the parameter-to-solution operator $S_{i}$, mapping a vector $p$ to some temperature matrix, i.e., some time and space discretization of $u$.

(c) Application of the observation operator to the solution of the parameter-to-solution operator.

(d) Solving (44) for simulated data $u_{\text {sim }}^{c, i}(i=1, \ldots, M)$ and comparison of the simulated material parameters $k_{\text {sim }}$ and $C_{\text {sim }}$ to the optimized functions $k_{\text {opt }}$ and $C_{\text {opt }}$.

5.1. Representation of Temperature-Dependent Material Parameters. The following paragraph only deals with the representation of the thermal conductivity $k(u)$ by some parameter vector $\underline{k} \in \mathbb{R}_{+}^{n}$, but the procedure works in the same way for the volumetric heat capacity $C(u)$.

First of all, one wants to fix the number $n>0$ of partition points $\left(u_{1}, \ldots, u_{n}\right)^{T} \in \mathbb{R}^{n}$ of the interval $U=\left[u_{\min }, u_{\max }\right]$. Setting the parameter vector $\underline{k}=\left(k_{1}, \ldots, k_{n}\right)^{T} \in \mathbb{R}_{+}^{n}$ of the corresponding function values and choosing an interpolation method, a function $k(u) \in \mathscr{C}^{1}(U)$ can be constructed, such that

$$
k\left(u_{i}\right)=k_{i},
$$

for $i=1, \ldots, n$

In this paper, the piecewise cubic hermite interpolating polynomials (PCHIPs) introduced in [25] is used, due to the favorable monotonic behavior of the interpolant with respect to the function values. Of course, one could also use an alternative interpolation method.

A big advantage in using an interpolation method rather than some representation as a linear combination of functions from a physically meaningful dictionary is that no a priori information about the functional form of the material parameters is needed. Additionally, adjusting one parameter $k_{i}$ of course leads to some changed interpolant $k(u)$, but only the function values in the local region of the corresponding $u_{i}$ are affected (Figure 5), which is very useful in the minimization process

$$
\min _{\underline{p} \in \mathbb{R}_{+}^{2 n}} J_{M}(\underline{p}) .
$$

Increasing the number $n$ of partition points allows the representation of more complex functions. Thus, for the estimation of the unknown material parameters $k(u)$ and $C(u)$, one wants to fix a sufficiently large number $n$ of partition points to determine enough function values $(\underline{k}, \underline{C})^{T}$ which fit the model and the observed data in an optimal way.

5.2. Implementation of the Parameter-To-Solution Operator. This section presents the implementation of the parameterto-solution operator $S$ which maps the parameter vector of 


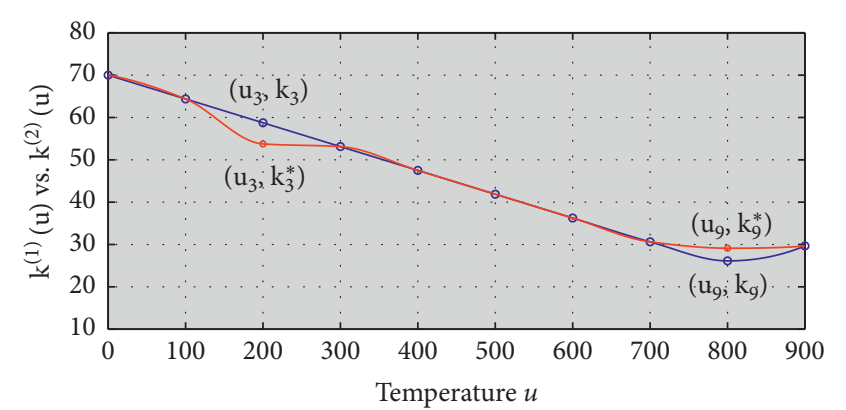

$$
\begin{array}{ll}
\circ\left(u_{i}, k_{i}\right), i=1, \ldots, 10 & - \text { Interpolant } k^{(1)}(u) \\
\text { - Variation of } k_{3} \text { and } k_{9} & - \text { Interpolant } k^{(2)}(u)
\end{array}
$$

FIGURE 5: Examples of PCHIP interpolants for $U=[0,900]$, $u_{i}=900 \cdot(i-1) /(n-1), i=1, \ldots, n=10$, with varying $k_{3}$ and. $k_{9}$.

function values $(\underline{k}, \underline{C})^{T}$ to a solution $u \in \mathscr{U}$ of the initialboundary-value problem (29)-(32). The parameter-to-solution operators $S_{i}$ are implemented analogously.

To get a solution of the IBVP numerically, a discretization of the temperature $u \in \mathscr{U}$ with respect to time $t \in I=[0, T]$ and the space variable $z \in \Omega=[0, L]$ is needed. For that reason, we define equidistant partitions

$$
\begin{aligned}
& 0=t_{1}<t_{2}<\cdots<t_{m}=T, \\
& 0=z_{1}<z_{2}<\cdots<z_{l}=L,
\end{aligned}
$$

for $I$ and $\Omega$ with increments $\Delta t=T / m-1$ and $\Delta z=L / l-1$, respectively. Here, $m, l>0$ are sufficiently large integers.

The output of $S$ can be interpreted as a temperature matrix $u \in \mathbb{R}_{+}^{m \times l}$ with components (Figure 6)

$$
\begin{gathered}
u_{i j}:=u\left(t_{i}, z_{j}\right), \quad i=1, \ldots, m, \\
j=1, \ldots, l,
\end{gathered}
$$

However, these components are yet to be determined from the IBVP. From the boundary conditions (30) and (31) and the initial temperature distribution (32), it holds that

$$
\begin{aligned}
& u_{i 1}=u^{b}\left(t_{i}\right), \quad i=1, \ldots m, \\
& u_{i l}=u^{t}\left(t_{i}\right), \quad i=1, \ldots m, \\
& u_{1 j}=u_{0}\left(z_{j}\right), \quad j=1, \ldots, l .
\end{aligned}
$$

Finally, one determines

$$
u_{i j} \text { for } i=2, \ldots, m \text { and } j=2, \ldots l-1,
$$

from (29) by using a classical finite difference method which yields the marching scheme

$$
u_{i j}=u_{i-1, j}+\frac{\Delta t}{(\Delta z)^{2} C\left(u_{i-1, j}\right)}\left(k_{i j}^{e} \cdot\left(u_{i-1, j+1}-u_{i-1, j}\right)-k_{i j}^{w} \cdot\left(u_{i-1, j}-u_{i-1, j-1}\right)\right)
$$

cf. Figure 6, with the harmonic means of thermal conductivities

$$
\begin{aligned}
& k_{i j}^{e}:=\frac{2 \cdot k\left(u_{i-1, j+1}\right) \cdot k\left(u_{i-1, j}\right)}{k\left(u_{i-1, j+1}\right)+k\left(u_{i-1, j}\right)}, \\
& k_{i j}^{w}:=\frac{2 \cdot k\left(u_{i-1, j+1}\right) \cdot k\left(u_{i-1, j}\right)}{k\left(u_{i-1, j+1}\right)+k\left(u_{i-1, j}\right)} .
\end{aligned}
$$

Note that an explicit scheme was chosen, i.e., the new time step component $u_{i j}$ is an explicit function of the old time step components $u_{i-1, j-1}, u_{i-1, j}$ and $u_{i-1, j+1}$. This way, by ensuring that the time and space discretizations are chosen carefully due to instability issues, the implementation of (59) is straightforward. One achieves overall good results by guaranteeing the stability condition

$$
\Delta t \leq \frac{(\Delta z)^{2}}{2 \max _{\widetilde{u} \in U} \lambda(\widetilde{u})}
$$

5.3. Application of the Observation Operator. Now that one interprets the output of $S$ as a temperature matrix $u \in \mathbb{R}_{+}^{m \times l}$, the observation operator $Q$ is modified by

$$
\begin{aligned}
Q: \mathbb{R}_{+}^{m \times l} & \longrightarrow \mathbb{R}_{+}^{m} \\
u & \longmapsto\left(u\left(t_{1}, \frac{L}{2}\right), \ldots, u\left(t_{m}, \frac{L}{2}\right)\right)^{T}
\end{aligned}
$$

Here, the partition of the time interval (51) is chosen to fit the data measurement setting, i.e., $u^{c} \in \mathbb{R}_{+}^{m}$ and $u_{i}^{c}$ is the core temperature measured at times $t_{i}$ for $i=1, \ldots, m$. Moreover, one requires the partition of the space interval (52) to contain the depth $z_{k}$ for some $1<k<l$ with $z_{k}=L / 2$, i.e., the space discretization yields a depth corresponding to the core of the thickness dimension of the heavy plate. Nevertheless, it is worth mentioning that it is possible to choose the partitions (51) and (52) in every possible way as long as the marching scheme (59) is numerically stable, cf. (61). In this case, however, it will be probably necessary to interpolate the temperature matrix $u$ such that one can extract a temperature vector

$$
\left(u\left(t_{1}, \frac{L}{2}\right), \ldots, u\left(t_{m}, \frac{L}{2}\right)\right)^{T}
$$

This way, given a full temperature matrix, the observation operator picks the temperature corresponding to the 


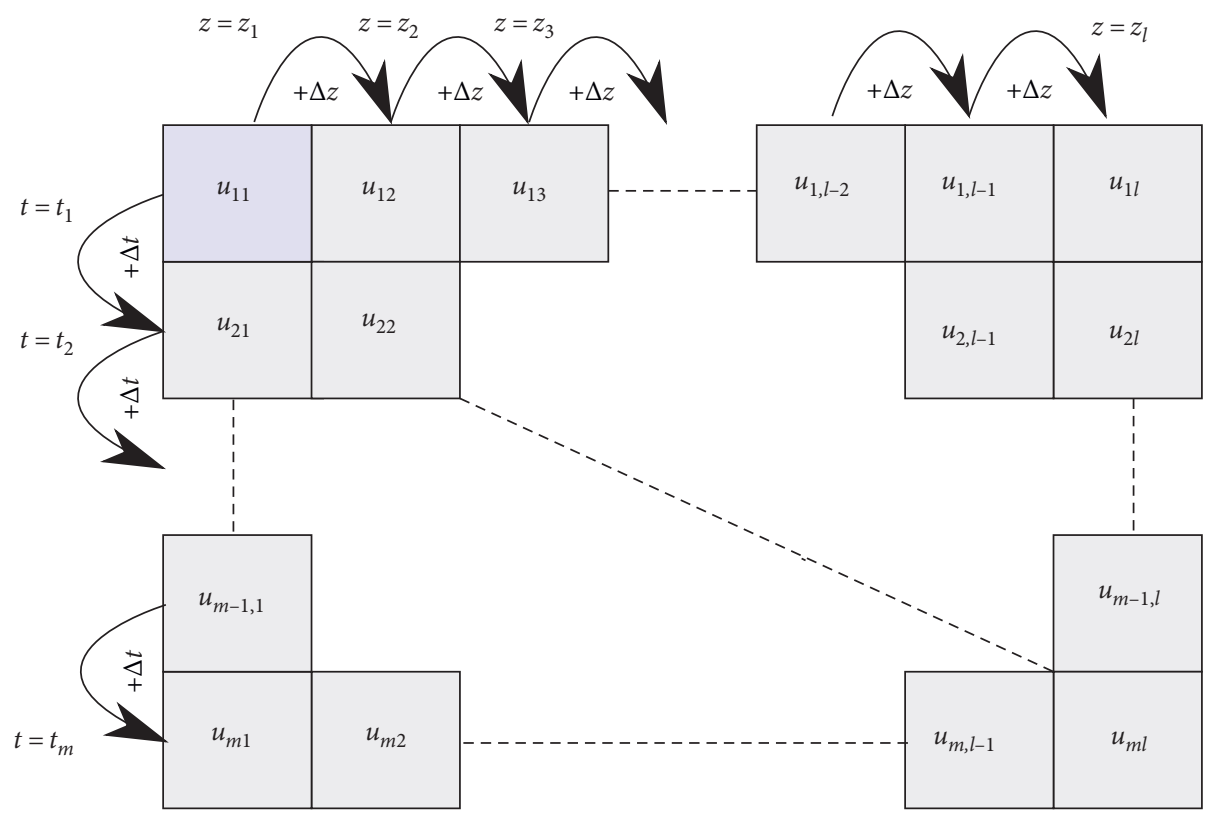

FIGURE 6: Illustration of the discretization of $u$.

core temperature at given time instances $t_{i}(i=1, \ldots, m)$ (Figure 7).

\subsection{Comparison of Simulated and Exact Material Parameters.} $k_{\text {sim }}$ and $C_{\text {sim }}$ In this part, the reliability of the implementation of (50) by means of simulations with synthetic data is demonstrated. Let $k_{\text {sim }}$ and $C_{\text {sim }}$ be some known (physically plausible) temperature-dependent material parameters from $\mathscr{C}^{1}(U)$. In particular, for temperatures, $u \in U=[0,900]$, let

$$
\begin{aligned}
& k_{\text {sim }}(u)=60-\frac{u}{30} \\
& C_{\text {sim }}(u)=7650 *\left(475+0.0265 u+0.000855 u^{2}-\frac{0.000855 u^{2}-0.1735 u+140}{1+e^{-0.1(u-700)}}\right),
\end{aligned}
$$

This represents a realistic setting which is oriented at the settings of [26] (Figure 8).

Inserting these functions into (45), the thermal conduction behavior of the material is prescribed. To simulate
$M=3$ experiments, three different triplets of boundary and initial temperatures are specified, i.e.,

$$
\left\{u^{b, 1}(t), u^{t, 1}(t), u_{0}^{1}(z)\right\},\left\{u^{b, 2}(t), u^{t, 2}(t), u_{0}^{2}(z)\right\} \text { and }\left\{u^{b, 3}(t), u^{t, 3}(t), u_{0}^{3}(z)\right\}
$$

Solving the associated IBVPs (45)-(48) numerically, one gets the temperature solution matrices $u_{i}$ for $i=1, \ldots, 3$, where one extracts the three core temperatures, respectively. To model a plausible measuring process, uniformly distributed noise is added to the data representing an appropriate maximum measurement precision error of the inserted thermocouples of $\pm 0.5^{\circ} \mathrm{C}$. Thus, the final data will consist of the three noisy core measurements $u^{c, 1}, u^{c, 2}$, and $u^{c, 3}$, reflecting the heat conduction behavior of the material parameters $k_{\text {sim }}, C_{\text {sim }} \in \mathscr{C}^{1}(U)$. Note that the components of the data vectors only lie in a subset $\widetilde{U} \in U=[0,900]$, which is called the observed temperature range interval.

The parameter vector of function values is subsequently determined as the solution of the least-squares problem 


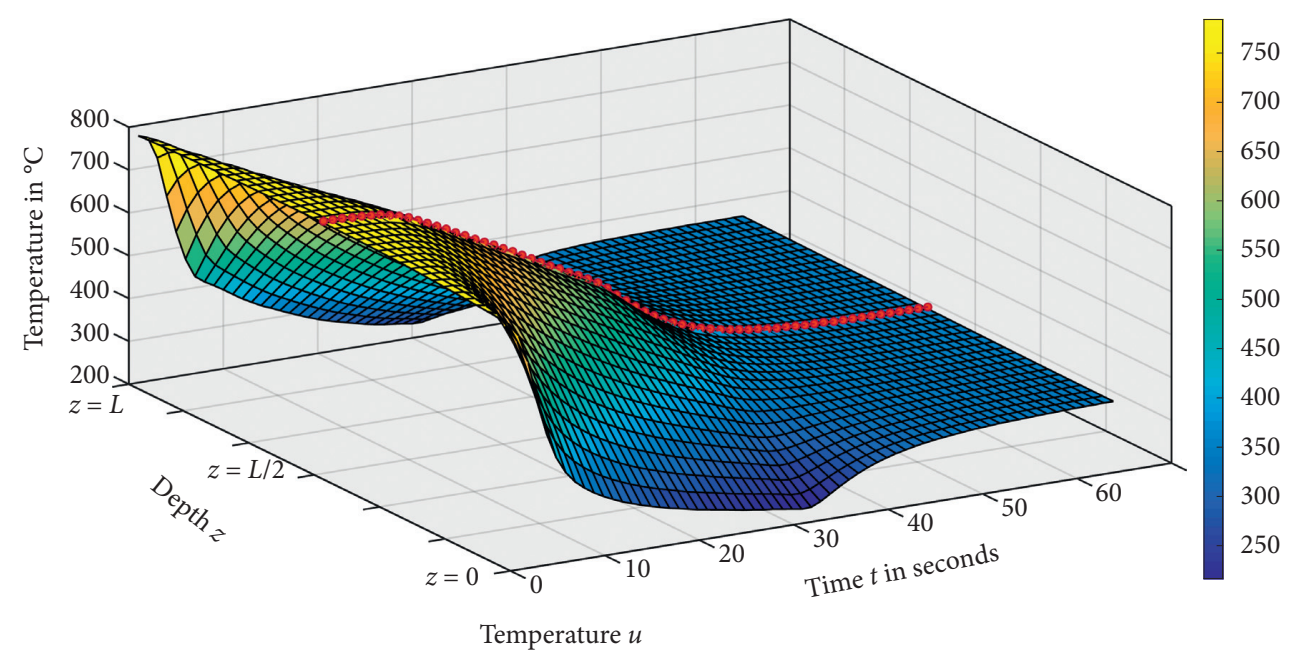

Figure 7: Observing core temperature at times $t_{i}$ (red line with red dots) from the interpolated temperature matrix $u$.

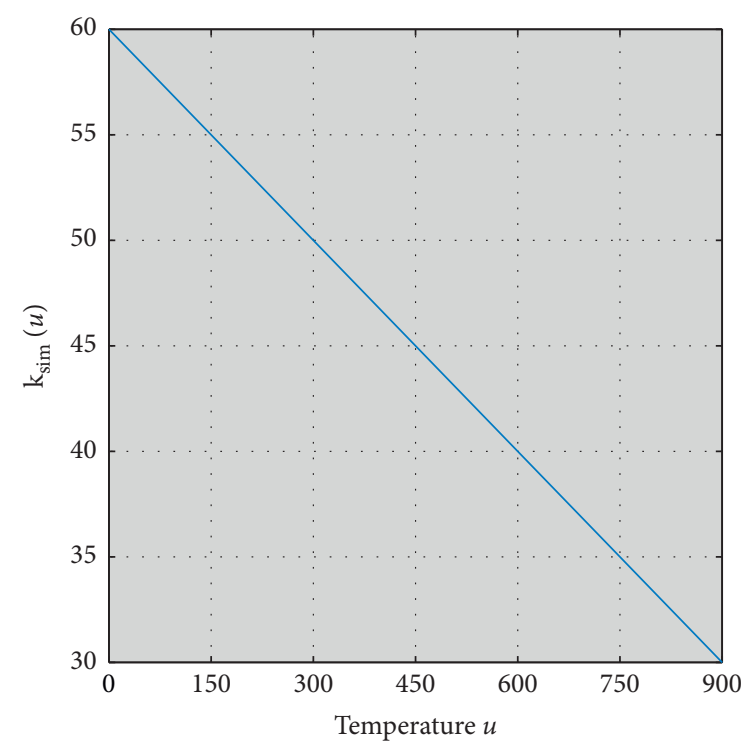

(a)

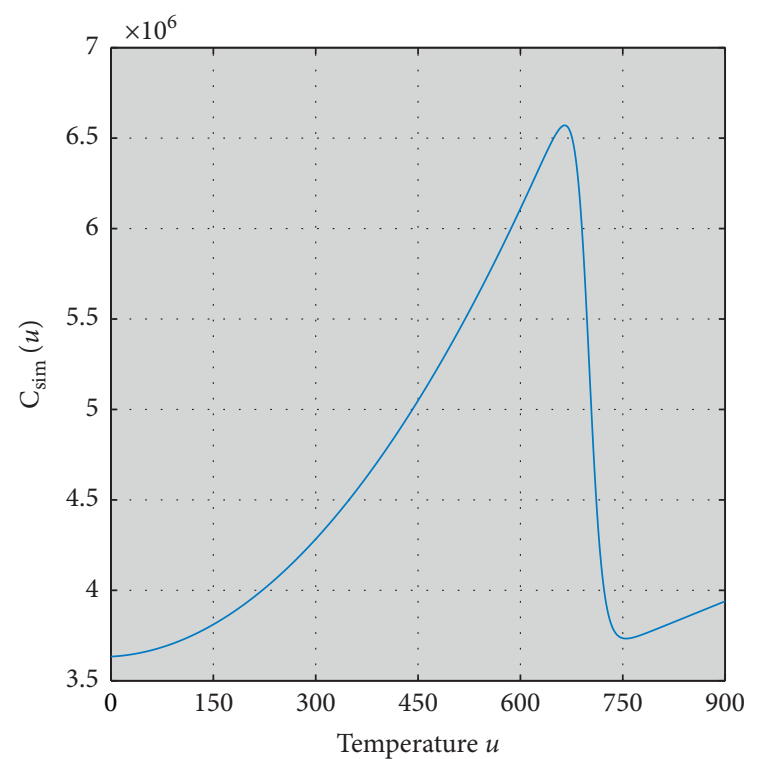

(b)

Figure 8: Plots of the simulated material parameters $k_{\text {sim }}$ and $C_{\text {sim }} \in \mathscr{C}^{1}(U)$.

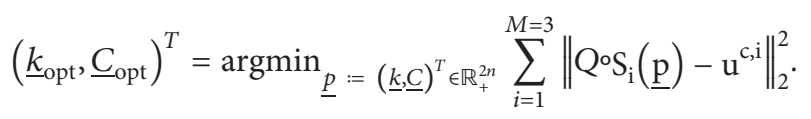

Corresponding to the fixed partition $\pi_{n}$ of $U$, here, $S_{i}$ maps a given parameter vector to the solution of (45)-(48), where $C(u)=C(u, p)$ and $k(u)=k(u, p)$ are the associated PCHIP interpolants. The boundary and initial temperatures are given in (66). The implementations of $S_{1}, S_{2}$, and $S_{3}$ are based on a marching scheme (59).

For the minimization of the objective functional in (67), one can use an iterative solver. In this paper, a trust-regionreflective algorithm of the subroutine lsqnonlin provided by the Matlab Optimization Toolbox was chosen [27].
To emphasize that no a priori information about the functional forms of the material parameters are needed, constant initial guesses are chosen, i.e.,

$$
\begin{aligned}
& k_{0}(u)=45, \\
& C_{0}(u)=4.5 * 10^{6},
\end{aligned}
$$

for all $u \in U$. Specifically, this means that $\mathbb{R}_{+}^{2 n} \ni p_{0}=\left(k_{0}, C_{0}\right)^{T}$, where

$$
\underline{k_{0}}=(\underbrace{45, \ldots, 45}_{n \text { times }})^{T} \text { and } \underline{C_{0}}=10^{6} *(\underbrace{4.5, \ldots, 4.5}_{n \text { times }})^{T} \text {, }
$$




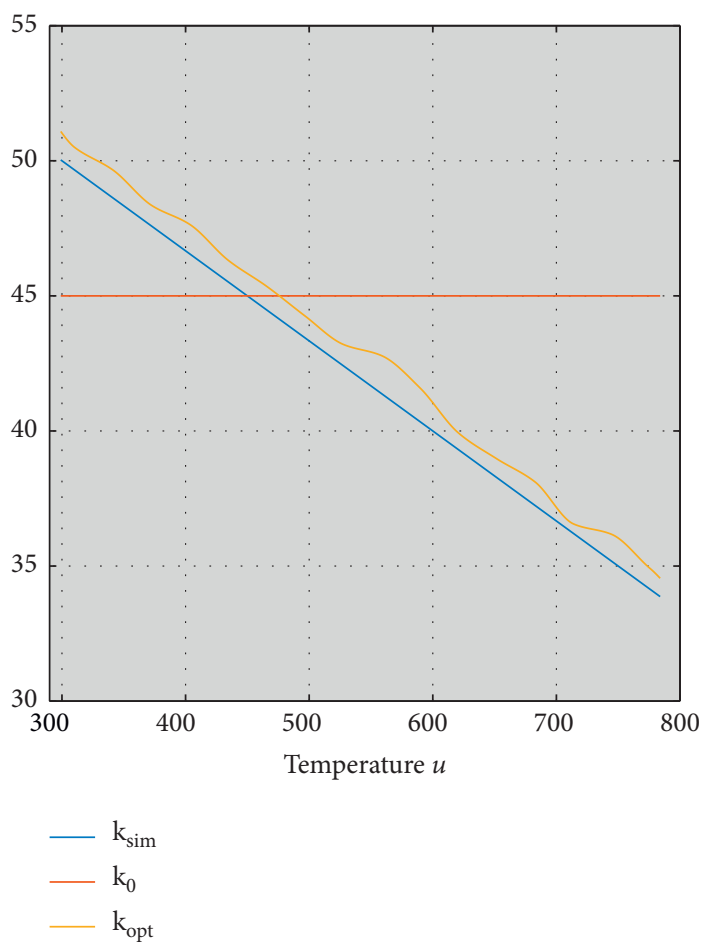

(a)

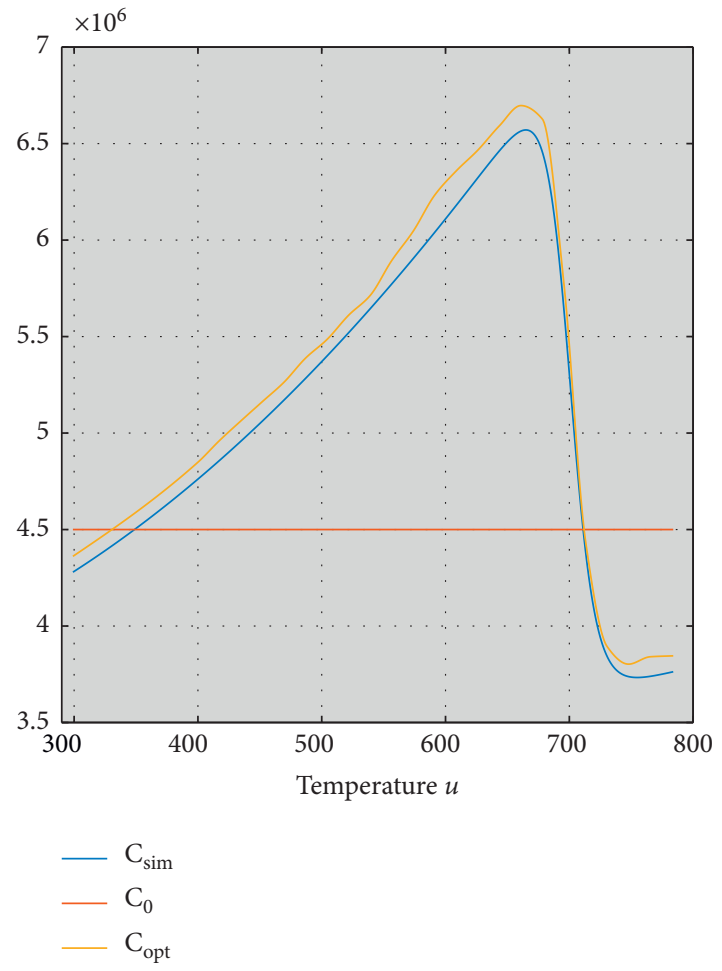

(b)

Figure 9: Comparison of exact (simulated), initial, and calculated (optimized) material parameters in $\tilde{U}$.

are the function values of the PCHIP interpolants $k_{0}(u)$ and $C_{0}(u)$ to the partition $\pi_{n}$ of $U$.

Implementing the parameter-to-solution operators $S_{i}$ and the observation operator $Q$, the task of finding (67) consists of a minimization in $\mathbb{R}_{+}^{2 n}$. The iterative solver tries to find a parameter vector $\left(\underline{k}_{\text {opt }}, \underline{C}_{\text {opt }}\right)^{T}$ by varying the function values and therefore the PCHIP interpolants.

The final results, i.e., the calculated PCHIP interpolants $k_{\text {opt }}(u)$ and $C_{\text {opt }}(u)$ to the initial guesses $k_{0}(u)$ and $C_{0}(u)$ are shown in Figure 9.

The small oscillations in $k_{\text {opt }}(u)$ and $C_{\text {opt }}(u)$ can be neglected and are most likely caused by the solver, i.e., the inherent stopping criteria of the minimizer. Nevertheless, the form of the functions are retained, but there are differences between $k_{\text {sim }}$ and $k_{\text {opt }}$ as well as between $C_{\text {sim }}$ and $C_{\text {opt }}$. This observation reinforces the fact that the solution of the inverse problem is not unique because the operators $F_{i}=$ $\mathrm{Q}^{\circ} \mathrm{S}_{\mathrm{i}}$ are not injective, cf. (37). As pointed out in Section 4.2, one can only expect to get a solution pair from a class of solutions having the same quotient. As a result, only the thermal diffusivities

$$
\lambda_{\text {sim }}=\frac{k_{\text {sim }}}{C_{\text {sim }}} \text { and } \lambda_{\text {opt }}=\frac{k_{\mathrm{opt}}}{C_{\mathrm{opt}}}
$$

can be compared. This yields an outcome that is illustrated in Figure 10 and shows an excellent performance of the method.

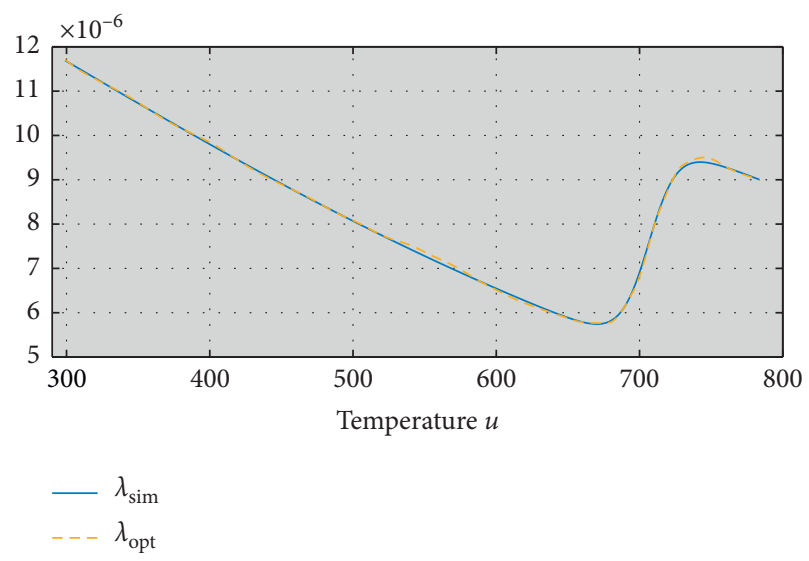

FIGURE 10: Comparison of exact (simulated) and calculated (optimized) thermal diffusivities.

\section{Conclusion}

The method described in this paper is able to numerically determine the temperature-dependent material parameters $k(u)$ and $C(u)$ (up to some canonical ambiguity) in a $1 \mathrm{D}$ nonlinear heat equation describing the heat conduction over the thickness of a cooled heavy plate. With the general interpolation procedure described in this paper, the identification process of general functions from an infinite-dimensional space $\mathscr{C}^{1}(U)$ turns over to a minimization in $\mathbb{R}_{+}^{2 n}$. 
Furthermore, by applying this parametrization approach, one is able to overcome the otherwise implicitly defined forward operator, which prevents a classical formulation of the IHCP. In the numerical part, although adding noise to the data, one can show that the conduction behavior of the material can be reconstructed almost perfectly in terms of the thermal diffusivity. Besides that, no a priori information about the functional shape of the material parameters is needed. Further investigations show that the method used in this paper is applicable to real data in the production of TMCP steel plates. There, the heat conduction determination provides physically plausible and valuable results in characterizing the underlying material, which is the first step in understanding and controlling the accelerated cooling (ACC) process. Future work is focused on modeling the heat fluxes on the surfaces depending on the surface temperature itself (Leidenfrost effect) and the control variables of the cooling device, i.e., the water load in every cooling zone and the feed rate of the heavy plate.

\section{Data Availability}

The data used to support the findings of this study are available from the corresponding author upon request.

\section{Conflicts of Interest}

The authors declare that they have no conflicts of interest.

\section{Acknowledgments}

Dimitri Rothermel and Thomas Schuster are indebted to the Dillinger Hüttenwerke AG for funding the research on this project.

\section{References}

[1] O. M. Alifanov, Inverse Heat Transfer Problems, Springer Science \& Business Media, Berlin, Germany, 2012.

[2] M. N. Ozisik, Inverse Heat Transfer: Fundamentals and Applications, CRC Press, Boca Raton, FL, USA, 2000.

[3] J. V. Beck and K. J. Arnold, Parameter Estimation in Engineering and Science, James Beck, London, UK, 1977.

[4] C. H. Huang and M. N. Özisik, "Inverse problem of determining unknown wall heat flux in laminar flow through a parallel plate duct," Numerical Heat Transfer, Part A: Applications, vol. 21, no. 1, pp. 55-70, 1992.

[5] Y.-C. Yang and W.-L. Chen, "A nonlinear inverse problem in estimating the heat flux of the disc in a disc brake system," Applied Thermal Engineering, vol. 31, no. 14-15, pp. 24392448, 2011.

[6] J. Su and G. F. Hewitt, "Inverse heat conduction problem of estimating time-varying heat transfer coefficient," Numerical Heat Transfer: Part A: Applications, vol. 45, no. 8, pp. 777-789, 2004.

[7] H. Kirsch, P. Flüss, W. Schütz, and A. Streisselberger, "New combinations of properties on heavy plate through the accelerated cooling process," Stahl und Eisen, vol. 119, no. 3, pp. 57-68, 1999, in German.

[8] Plate Mills, "Mulpic interrupted cooling provides unique advantages for plate mills," Metals Magazine 2/ 2014-Technology, Plate Mills, , pp. 46-47, Rourkela, Odisha.
[9] Y. Serizawa, S. Nakagawa, Y. Kadoya et al., "Plate cooling technology for the thermo mechanical control process (TMCP) in Nippon steel \& Sumitomo Metal Corporation," Nippon Steel \& Sumitomo Metal Technical Report, vol. 110, pp. 17-24, 2015.

[10] S. Nakagawa, H. Tachibana, Y. Kadoya et al., "Cooling control technology for steel plates in Kashima Steel Works," in Proceedings of the IFAC Workshop on Future Trends in Automation of the Mineral and Metal Processing, pp. 193-197, Finland, Europe, August 2012.

[11] N. Karwa, Experimental Study of Water Jet Impingement Cooling of Hot Steel Plates, Ph.D. thesis, TUprints, Moscow, Russia, 2012.

[12] G. Ben-yu and J. Zou, "An augmented Lagrangian method for parameter identifications in parabolic systems," Journal of Mathematical Analysis and Applications, vol. 263, no. 1, pp. 49-68, 2001.

[13] H. W. Engl and J. Zou, "A new approach to convergence rate analysis of Tikhonov regularization for parameter identification in heat conduction," Inverse Problems, vol. 16, no. 6, p. 1907, 2000.

[14] M. S. Hussein, D. Lesnic, and M. I. Ivanchov, "Simultaneous determination of time-dependent coefficients in the heat equation," Computers \& Mathematics with Applications, vol. 67, no. 5, pp. 1065-1091, 2014.

[15] K. Kunisch and G. Peichl, "Estimation of a temporally and spatially varying diffusion coefficient in a parabolic system by an augmented Lagrangian technique," Numerische Mathematik, vol. 59, no. 1, pp. 473-509, 1991.

[16] M. Cui, X. Gao, and J. Zhang, "A new approach for the estimation of temperature-dependent thermal properties by solving transient inverse heat conduction problems," International Journal of Thermal Sciences, vol. 58, pp. 113-119, 2012.

[17] M. Mierzwiczak and J. A. Kołodziej, "The determination temperature-dependent thermal conductivity as inverse steady heat conduction problem," International Journal of Heat and Mass Transfer, vol. 54, no. 4, pp. 790-796, 2011.

[18] C.-H. Huang and Y. Jan-Yuan, "An inverse problem in simultaneously measuring temperature-dependent thermal conductivity and heat capacity," International Journal of Heat and Mass Transfer, vol. 38, no. 18, pp. 3433-3441, 1995.

[19] H. W. Engl, M. Hanke, and A. Neubauer, "Regularization of inverse problems," Springer Science \& Business Media, vol. $375,1996$.

[20] A. K. Louis, Inverse and Ill-Posed Problems, Springer-Verlag, Berlin, Germany, 2013.

[21] W. Schütz, H. J. Kirsch, P. Flüss, and V. Schwinn, "Extended property combinations in thermomechanically control processed steel plates by application of advanced rolling and cooling technology," Ironmaking \& Steelmaking, vol. 28, no. 2, pp. 180-184, 2001.

[22] X.-L. Tian, W. Yu, and Q.-J. Song, "Practice of using mulpic cooling device in development of high grade steel," Iron \& Steel, vol. 5, 2009.

[23] T. Roubíček, Nonlinear Partial Differential Equations with Applications, Vol. 153, Springer Science \& Business Media, Berlin, Germany, 2013.

[24] T. Schuster, B. Kaltenbacher, B. Hofmann, and K. S. Kazimierski, "Regularization methods in Banach spaces," Walter de Gruyter, vol. 10, 2012.

[25] F. N. Fritsch and R. E. Carlson, "Monotone piecewise cubic interpolation," SIAM Journal on Numerical Analysis, vol. 17, no. 2, pp. 238-246, 1980. 
[26] K. Harste, Untersuchungen zur Schrumpfung und zur Entstehung von mechanischen Spannungen während der Erstarrung und nachfolgender Abkühlung zylindrischer Blöcke aus $\mathrm{Fe}$-C-Legierungen, Ph.D. thesis, Technische Universität Clausthal, Clausthal-Zellerfeld, LS, Germany, 1989.

[27] The MathWorks, Matlab Optimization Toolbox 8.0, The MathWorks, Natick, MA, USA, 1990. 